\title{
Asymptotic Behavior of Eigenvalues of Toeplitz Forms*
}

\section{ROSENBLATT}

1. Introduction. Let $F(\lambda)=\left\{F_{j k}(\lambda) ; j, k=1, \cdots, s\right\}$ be an $s \times s$ matrixvalued function of bounded variation on $[-\pi, \pi]$. By this we mean that every complex-valued element $F_{j k}(\lambda)$ is of bounded variation. Further, let every difference $F\left(\lambda_{1}\right)-F\left(\lambda_{2}\right)$ be Hermitian. It will be convenient and in no way less general to take $F(-\pi)=0$, the null matrix. Let

$$
r_{k}(d F)=\frac{1}{2 \pi} \int_{-\pi}^{\pi} e^{i k \lambda} d F(\lambda), \quad k=0, \pm 1, \cdots,
$$

be the sequence of Fourier-Stieltjes coefficients of $F$. The sequence $r_{k}(d F)$ is a sequence of $s \times s$ matrices. Let the Hermitian matrix

$$
R_{n}\left(d F^{\prime}\right)=\left\{r_{i-k}(d F) ; j, k=1, \cdots, n\right\}
$$

be the $n s \times n s$ block Toeplitz matrix generated by $d F$. Since $F$ is of bounded variation, its derivative

$$
f(\lambda)=\frac{d F(\lambda)}{d \lambda}
$$

exists almost everywhere and is Hermitian. Let $\mu_{1}(\lambda) \leqq \mu_{2}(\lambda) \leqq \cdots \leqq \mu_{s}(\lambda)$ be the eigenvalues of $f(\lambda)$ in order of magnitude. The functions $\mu_{j}(\lambda), j=1, \cdots, s$, are measurable (see Grires [3]). Let $\mu_{i, n}, j=1, \cdots, n s$, be the eigenvalues of $R_{n}\left(d F^{\prime}\right)$ and $N_{n}(x)$ the number of eigenvalues of $R_{n}\left(d F^{\prime}\right)$ less than or equal to $x$. We show that

$$
\lim _{n \rightarrow \infty} \frac{N_{n}(x)}{n s}=\frac{1}{2 \pi s} \sum_{j=1}^{s} m\left\{\lambda \mid \mu_{j}(\lambda) \leqq x\right\}
$$

at every point of continuity of the right hand side, where $m$ is Lebesgue measure on $[-\pi, \pi]$. The case of Toeplitz matrices arises when $s=1$ and was treated by Szegö and Grenander \& Szegö (see [2]). The result obtained in this paper is a generalization of that given by Gyires [3] and Rosenblatt [6] for block Toeplitz matrices.

* The results presented in this paper were obtained in the course of research carried out under grant NSF-G19046 of the National Science Foundation. 
A crude upper bound is obtained for the maximal eigenvalue of Toeplitz matrices generated by an absolutely continuous $F$. This upper bound is used to obtain a result on the asymptotic distribution of covariance estimates of normal stationary processes.

2. Nondecreasing $F(\lambda)$. We first prove the result on the asymptotic distribution of eigenvalues of block Toeplitz forms in the case of nondecreasing $F(\lambda)$. The function $F(\lambda)$ is said to be nondecreasing if $F\left(\lambda_{1}\right)-F\left(\lambda_{2}\right)$ is a positive definite matrix whenever $\lambda_{1} \geqq \lambda_{2}$.

Lemma 1. Let $F(\lambda)$ be an $s \times s$ nondecreasing matrix-valued function of bounded variation on $[-\pi, \pi]$. The limiting distribution of the eigenvalues of the block Toeplitz matrix $R_{n}(d F)$ as $n \rightarrow \infty$ is given by

$$
\frac{1}{2 \pi s} \sum_{i=1}^{s} m\left\{\lambda \mid \mu_{j}(\lambda) \leqq x\right\} .
$$

Let $G_{n}(x)$ be the distribution function of the eigenvalues of $R_{n}(d F)$, that is,

$$
G_{n}(x)=\frac{N_{n}(x)}{s n} .
$$

We need only consider the distribution functions $G_{n}(x)$ on $[0, \infty)$. Now

$$
\int_{0}^{\infty} x d G_{n}(x)=\frac{1}{n s} \sum_{i=1}^{n s} \mu_{i, n}=\frac{1}{n s} \mathrm{sp}\left(R_{n}\left(d F^{\prime}\right)\right)=\frac{1}{s} \mathrm{sp}\left(r_{0}\left(d F^{\prime}\right)\right)
$$

where sp $(M)$ denotes the trace of the matrix $M$. Notice that

$$
y\left[1-G_{n}(y)\right] \leqq \int_{y}^{\infty} x d G_{n}(x) \leqq \frac{1}{s} \mathrm{sp}\left(r_{0}\right)=c,
$$

so that

$$
1-G_{n}(y) \leqq \frac{c}{y}
$$

Consider any weak limit $G(y)$ of a subsequence of the sequence $G_{n}(y)$. Clearly any such limit must also satisfy

$$
1-G(y) \leqq \frac{c}{y}
$$

and hence is a distribution function. Let $z$ be a nonnegative number. The basic result on the determinant of the prediction error covariance matrix in the multidimensional prediction problem tells us that

$$
\begin{aligned}
& \frac{1}{n s} \sum_{i=1}^{n s} \log \left(1+z \mu_{i, n}\right)=\int_{0}^{\infty} \log (1+z x) d G_{n}(x) \\
& \quad \rightarrow \frac{1}{2 \pi s} \int_{-\pi}^{\pi} \log \operatorname{det}(I+z f(\lambda)) d \lambda=\frac{1}{2 \pi s} \sum_{j=1}^{s} \int_{-\pi}^{\pi} \log \left(1+z \mu_{j}(\lambda)\right) d \lambda
\end{aligned}
$$


as $n \rightarrow \infty$ (see [5] and [6]). On integrating by parts we see that

$$
\begin{aligned}
\int_{0}^{\infty} \log (1+z x) d G_{n}(x)=\left.\log (1+z x)\left(G_{n}(x)-1\right)\right|_{0} ^{\infty} \\
\quad+\int_{0}^{\infty} \frac{z}{1+z x}\left(1-G_{n}(x)\right) d x=\int_{0}^{\infty} \frac{z}{1+z x}\left(1-G_{n}(x)\right) d x .
\end{aligned}
$$

Relations (11) and (12) imply that for any weak limit $G(y)$ of a subsequence of $G_{n}(y)$

$$
\int_{0}^{\infty} \frac{z}{1+z x}(1-G(x)) d x=\frac{1}{2 \pi s} \sum_{i=1}^{s} \int_{-\pi}^{\pi} \log \left(1+z \mu_{j}(\lambda)\right) d \lambda .
$$

The distribution function (5) obviously satisfies (13). To complete the proof for nondecreasing $F$ we only have to show that $G$ is uniquely determined by the transform (13). Consider the uniqueness problem for the transform

$$
\int_{0}^{\infty} \frac{z}{1+z x} H(x) d x, \quad z \geqq 0,
$$

where $H$ is of bounded variation and satisfies $|H(x)| \leqq k /(1+x)$ for some constant $k$. This is equivalent to the uniqueness problem for the Stieltjes transform

$$
\int_{0}^{\infty} \frac{1}{z+x} H(x) d x, \quad z>0 .
$$

A proof of uniqueness is easily given. Suppose

$$
\int_{0}^{\infty} \frac{1}{z+x} H(x) d x \equiv 0
$$

for all $z>0$. Equation (16) can be rewritten as

$$
\int_{0}^{\infty} e^{-z u} \int_{0}^{\infty} e^{-x u} H(x) d x d u \equiv 0 .
$$

But this implies that

$$
\int_{0}^{\infty} e^{-x u} H(x) d x \equiv 0 .
$$

It follows that $H(x) \equiv 0$ almost everywhere. The proof of Lemma 1 is complete.

It is readily seen that the conclusion of Lemma 1 holds for every $F$ of bounded variation such that $F(\lambda)+a(\lambda+\pi) I$ is nondecreasing for some real value $a$. It similarly holds for every $F$ of bounded variation such that $F(\lambda)+a(\lambda+\pi) I$ is nonincreasing for some real value $a$.

3. General $F$. In this section it is shown that the conclusion of Lemma 1 holds for general Hermitian $F$ of bounded variation. The extension will depend on the following remarks. Given two $n \times n$ Hermitian matrices $A, B$ with 
$A-B \geqq 0$ (difference positive definite), the eigenvalues $\lambda_{i}$ and $\mu_{i}$ of $A$ and $B$ respectively (assume the eigenvalues are indexed in order of magnitude) satisfy the corresponding inequalities, that is,

$$
\lambda_{i} \geqq \mu_{i}, \quad j=1, \cdots, n .
$$

This follows readily from the minimax property of eigenvalues (see HILBERT \& Courant [1]).

Theorem 1. Let $F(\lambda)(F(-\pi)=0)$ be an $s \times s$ Hermitian matrix-valued function of bounded variation on $[-\pi, \pi]$. The limiting distribution of the eigenvalues of the block Toeplitz matrix $R_{n}(d F)$ as $n \rightarrow \infty$ is given by

$$
\frac{1}{2 \pi s} \sum_{j=1}^{s} m\left\{\lambda \mid \mu_{j}(\lambda) \leqq x\right\} .
$$

We first prove the desired result for such a function $F$ whose singular part is nondecreasing. Let $f(\lambda)=d F(\lambda) / d \lambda$. The singular part of $F$ is given by $F(\lambda)-\int_{-\pi}^{\lambda} f(\mu) d \mu$. Let

$$
S_{a}=\{\lambda \mid f(\lambda)+a I \geqq 0\},
$$

and $\bar{S}_{a}$ be the complement of $S_{a}$. The sets $S_{a}$ are nondecreasing as $a \rightarrow \infty$ and such that the Lebesgue measure $m\left(S_{a}\right) \rightarrow 2 \pi$ as $a \rightarrow \infty$. This follows from the fact that $f(\lambda)$ is integrable, that is, every element of $f$ is integrable. Given any value $\lambda$, let

$$
f^{+}(\lambda)=\sum_{u, v=1}^{s} s\left|f_{u, v}(\lambda)\right| \cdot I .
$$

Note that

$$
f(\lambda) \leqq f^{+}(\lambda)
$$

( $A \geqq B$ means that $A-B \geqq 0$ ). Inequality (22) is to be understood as holding almost everywhere where the elements of the matrices are finite and hence well defined. Let

$$
F^{a}(\lambda)=F(\lambda)-\int_{-\pi}^{\lambda} f(\mu) d \mu+\int_{-\pi}^{\lambda} f^{a}(\mu) d \mu
$$

where

$$
f^{a}(\lambda)=\left\{\begin{array}{ccc}
f(\lambda) & \text { on } & S_{a} \\
f^{+}(\lambda) & \text { on } & \overline{S_{a}} .
\end{array}\right.
$$

Because of (22) it follows that

$$
R_{n}(d F) \leqq R_{n}\left(d F^{a}\right)
$$

for all $a$. Further, $R_{n}\left(d F^{a}\right)$ is nonincreasing as $a \rightarrow \infty$. Let $G^{(n)}(x), G_{a}^{(n)}(x)$ be the distribution functions of the eigenvalues of $R_{n}(d F)$ and $R_{n}\left(d F^{a}\right)$ respectively. 
It then follows that

$$
G_{a}^{(n)}(x) \leqq G^{(n)}(x)
$$

for all $n, a$, and $x$. The integrability of $f(\lambda), f^{+}(\lambda)$, inequality (25), and the fact that $m\left(\bar{S}_{a}\right) \rightarrow 0$ as $a \rightarrow \infty$ imply that for every $\epsilon>0$ there is an $a(\epsilon)>0$ such that for $a>a(\epsilon)$

$$
\begin{aligned}
& 0 \leqq \frac{1}{s n} \mathrm{sp}\left(R_{n}\left(d F^{a}\right)-R_{n}(d F)\right) \\
&=\int_{-\infty}^{\infty} x d\left[G_{a}^{(n)}(x)-G^{(n)}(x)\right]=\int_{-\infty}^{\infty}\left[G^{(n)}(x)-G_{a}^{(n)}(x)\right] d x<\epsilon
\end{aligned}
$$

independently of $n$. By the remarks at the end of section 2, it follows that $G_{a}^{(n)}(x)$ has the limiting distribution

$$
G_{a}(x)=\frac{1}{2 \pi s} \sum_{j=1}^{s} m\left\{\lambda \mid \mu_{i}^{a}(\lambda) \leqq x\right\}
$$

as $n \rightarrow \infty$ where the $\mu_{i}^{a}(\lambda)$ are the eigenvalues of $f^{a}(\lambda)$. Let $H(x)$ be the limit of any subsequence of the functions $G^{(n)}(x)$. It follows that

$$
G_{a}(x) \leqq H(x)
$$

for all $x$ and $a$. Inequality (29) implies that

$$
0 \leqq \int_{-\infty}^{\infty}\left[H(x)-G_{a}(x)\right] d x \leqq \epsilon
$$

for all $a>a(\epsilon)$. The family of functions $G_{a}(x)$ is nondecreasing at each $x$ as $a \rightarrow \infty$ and has as limit as $a \rightarrow \infty$

$$
G(x)=\frac{1}{2 \pi s} \sum_{i=1}^{s} m\left\{\lambda \mid \mu_{i}(\lambda) \leqq x\right\} .
$$

Thus

$$
H(x) \geqq G(x)
$$

and

$$
0 \leqq \int_{-\infty}^{\infty}[H(x)-G(x)] d x \leqq \epsilon
$$

for every $\epsilon$. But this implies that $H(x)=G(x)$. Since this is true for the limit $H(x)$ of any subsequence of $G^{(n)}(x)$, there is a limiting distribution which is given by $G(x)$. The proof is complete for $F$ with a nondecreasing singular part.

We now complete the proof of Theorem 1 by showing that (5) is the asymptotic distribution of eigenvalues of $R_{n}(d F)$ for a general Hermitian $F$ of bounded variation. The singular part of $F$ is

$$
H(\lambda)=F(\lambda)-\int_{-\pi}^{\lambda} f(\mu) d \mu
$$


where $f(\lambda)=d F(\lambda) / d \lambda$. Let

$$
H^{+}(\lambda)=\sum_{u, v=1}^{s} s \int_{-\pi}^{\lambda}\left|d H_{u, v}(\mu)\right| \cdot I
$$

Set

$$
F_{1}(\lambda)=\int_{-\pi}^{\lambda} f(\mu) d \mu+H^{+}(\lambda), \quad F_{2}(\lambda)=\int_{-\pi}^{\lambda} f(\mu) d \mu-H^{+}(\lambda) .
$$

$F_{1}$ has a nondecreasing singular part $H^{+}$and $F_{2}$ a nonincreasing singular part $-H^{+}$. Further,

$$
R_{n}\left(d F_{1}\right) \geqq R_{n}(d F) \geqq R_{n}\left(d F_{2}\right)
$$

for all $n$. Since $R_{n}\left(d F_{1}\right), R_{n}\left(d F_{2}\right)$ have the same asymptotic eigenvalue distribution (5) by the result on the asymptotic distribution of eigenvalues for a function with a nondecreasing singular part given in the last paragraph, it follows from (37) that $R_{n}(d F)$ has the asymptotic eigenvalue distribution (5).

4. Asymptotic distribution of covariance estimates. It will be useful to have an upper bound on the maximal eigenvalue of the ordinary Toeplitz matrix $R_{n}(f)$ generated by a nonnegative function $f \varepsilon L^{2}$ in our derivation of the asymptotic distribution of the covariance estimates of a normal stationary process. There are refined estimates of $\lambda_{n, n}$ when $f$ is a bounded function satisfying certain regularity conditions (see [2]). However, we wish to allow $f$ to be unbounded. Let

$$
G(x)=\frac{1}{2 \pi} m\{\lambda \mid f(\lambda) \leqq x\} .
$$

Lemma 2. The maximal eigenvalue $\lambda_{n, n}$ of the Toeplitz matrix $R_{n}(f)$ generated by $f \varepsilon L$ is bounded above by

$$
n \int_{b(n)}^{\infty} x d G(x)
$$

where $b(n)$ is the supremum of the values $\alpha$ such that $1-G(\alpha)>1 / n$. If $f \varepsilon L^{2}$, this upper bound is of smaller order than $n^{\frac{1}{2}}$ as $n \rightarrow \infty$.

The eigenvalue $\lambda_{n, n}$ is the maximal value attained by the quadratic form $c R_{n} c^{\prime}$ with $c$ an $n$-vector subject to the restraint $c c^{\prime}=1$. But

$$
c R_{n} c^{\prime}=\frac{1}{2 \pi} \int_{-\pi}^{\pi}\left|c_{n}\left(e^{i \lambda}\right)\right|^{2} f(\lambda) d \lambda, \quad c_{n}\left(e^{i \lambda}\right)=\sum_{j=1}^{n} c_{j} e^{i j \lambda}
$$

and the absolute maximum of $\left|c_{n}\left(e^{i \lambda}\right)\right|^{2}$ subject to the restraint $c c^{\prime}=1$ is $n$. Let

$$
S_{x}=\{\lambda \mid f(\lambda) \geqq x\}
$$

We certainly overestimate $\lambda_{n, n}$ by replacing $\left|c_{n}\left(e^{i \lambda}\right)\right|^{2}$ by a function equal to $n$ on the set $S_{b(n)}$. Now 


$$
\frac{1}{2 \pi} n \int_{S_{b(n)}} f(\lambda) d \lambda=n \int_{b(n)}^{\infty} x d G(x) .
$$

If $f \varepsilon L^{2}$ then $\int x^{2} d G(x)<\infty$ and by the Schwarz inequality

$$
n \int_{b(n)}^{\infty} x d G(x) \leqq n\left[\int_{b(n)}^{\infty} d G(x) \int_{b(n)}^{\infty} x^{2} d G(x)\right]^{1 / 2}=o\left(n^{1 / 2}\right) .
$$

An upper bound for the maximal eigenvalue of a block Toeplitz matrix can be obtained by similar techniques.

We now consider the asymptotic distribution of the estimates

$$
r_{k}^{*}=\frac{1}{N} \sum_{j=1}^{N-|k|} X_{i} X_{i+k}, \quad k=0,1, \cdots, s,
$$

of the covariances $r_{k}, k=0,1, \cdots, s$, of a normal stationary process $\left\{X_{k}\right\}$.

Theorem 2. Let $\left\{X_{k} ; k=0, \pm 1, \cdots\right\}$ be a normal stationary process with mean $\mathrm{E} X_{k} \equiv 0$ and covariances

$$
r_{k}=\operatorname{cov}\left(X_{j}, X_{j+k}\right)=\frac{1}{2 \pi} \int_{-\pi}^{\pi} e^{i k \lambda} f(\lambda) d \lambda \quad k=0, \pm 1, \cdots,
$$

with $f \varepsilon L^{2}$. Then

$$
\sqrt{N}\left(r_{k}^{*}-\mathrm{E} r_{k}^{*}\right), \quad k=0,1, \cdots, s,
$$

are asymptotically jointly normally distributed with mean zero and covariances

$$
c_{j, k}=\frac{1}{\pi} \int_{-\pi}^{\pi} \cos j \lambda \cos k \lambda f^{2}(\lambda) d \lambda, \quad j, k=0,1, \cdots, s .
$$

The joint characteristic function of the random variables (43) is

$$
\begin{aligned}
\mathrm{E}\left(\operatorname { e x p } \left\{\sum _ { k = 0 } ^ { s } i t _ { k } \left(r_{k}^{*}-\right.\right.\right. & \left.\left.\left.\mathrm{E} r_{k}^{*}\right) \sqrt{N}\right\}\right) \\
= & \exp \left\{-\sum_{k=0}^{s} i t_{k} \sqrt{N} \mathrm{E}_{k}^{*}\right\}(2 \pi)^{-1 / 2 N}\left|R_{N}\right|^{-1 / 2} \\
& \cdot \int_{-\infty}^{\infty} \cdots \int \exp \left\{i x T_{N} x^{\prime} / \sqrt{N}-\frac{1}{2} x R_{N}^{-1} x^{\prime}\right\} d x \\
= & \left|I-2 i R_{N} T_{N} / \sqrt{N}\right|^{-1 / 2} \exp \left\{-\sum_{k=0}^{s} i t_{k} v \bar{N} \mathrm{E} r_{k}^{*}\right\}
\end{aligned}
$$

where $x$ is a row $N$-vector, $R_{N}=R_{N}(f)$, and $T_{N}=\left\{t_{i, k} ; j, k=1, \cdots, N\right\}$ is the Toeplitz matrix with $t_{i, i} \equiv t_{0}, t_{i, i \neq k}=\frac{1}{2} t_{|k|}$ if $0<k \leqq s$, and all other elements zero. Now expression (45) can be written as

$$
\exp \left\{-\frac{1}{2} \sum_{j=1}^{N}\left[\log \left(1-2 i \mu_{i, N} / \sqrt{N}\right)+2 i_{\mu_{i, N}} / \sqrt{N}\right]\right\}
$$

where the $\mu_{j, N}$ are the eigenvalues of $R_{N}^{1 / 2} T_{N} R_{N}^{1 / 2}$. Here $R_{N}^{\frac{1}{2}}$ is the positive definite 
square root of $R_{N}$. However,

$$
\mu_{i, N}=O\left(\max _{i} \alpha_{i, N} \sum_{j}\left|t_{i}\right|\right),
$$

where the $\alpha_{i, N}$ are the eigenvalues of $R_{N}$. By Lemma 2 the eigenvalues of $R_{N}$ are $o\left(N^{\frac{1}{2}}\right)$ uniformly in $j$ so that $\mu_{j, N} / \sqrt{N}=o(1)$ uniformly in $j$. Therefore (45) can be given as

$$
\exp \left\{-\operatorname{sp}\left(R_{N} T_{N}\right)^{2} / N+O\left(\sum_{i=1}^{N}\left|\mu_{i, N}\right|^{3} / N^{3 / 2}\right)\right\} .
$$

But

$$
\sum\left|\mu_{i, N}\right|^{3} \leqq\left(\sum \mu_{i, N}^{2} \sum \mu_{j, N}^{4}\right)^{1 / 2}=\left[\operatorname{sp}\left(R_{N} T_{N}\right)^{2} \operatorname{sp}\left(R_{N} T_{N}\right)^{4}\right]^{1 / 2} .
$$

Furthermore

$$
0 \leqq \mathrm{sp}\left(R_{N} T_{N}\right)^{2 k} \leqq \mathrm{sp}\left(M_{N}\right)^{2 k}, \quad k=1,2, \cdots,
$$

where $M_{N}$ is the $N \times N$ Toeplitz matrix with elements

$$
m_{i-j}=\sum_{|u| \leqq s}\left|r_{i-j-u}\right| \cdot\left|t_{u}\right| .
$$

Notice that $M_{N}$ is generated by a real-valued function $g \varepsilon L^{2}$ since $m_{k}=m_{-k}$ and $\sum\left|r_{k}\right|^{2}<\infty$. The fact that $\sum m_{k}^{2}<\infty$ implies that sp $\left(M_{N}^{2}\right)=O(N)$. Since $g$ \& $L^{2}$, Lemma 2 indicates that the eigenvalues $\eta_{i, N}$ of $M_{N}$ are all uniformly $o\left(N^{\frac{1}{3}}\right)$. But then

$$
\operatorname{sp}\left(M_{N}\right)^{4}=\sum \eta_{i, N}^{4}=o(N) \operatorname{sp}\left(M_{N}\right)^{2}=o\left(N^{2}\right) .
$$

This implies that

$$
\sum_{i=1}^{N}\left|\mu_{i, N}\right|^{3} / N^{3 / 2}=o(1)
$$

as $N \rightarrow \infty$. A simple computation indicates that

$$
\begin{aligned}
& \lim _{N \rightarrow \infty} \frac{1}{N} \operatorname{sp}\left(R_{N} T_{N}\right)^{2}=\sum_{i, k=-8}^{s} \frac{1}{4}\left(1+\delta_{i, 0}\right)\left(1+\delta_{k, 0}\right) t_{|j|} t_{|k|} \frac{1}{2 \pi} \\
& \cdot \int_{-\pi}^{\pi} e^{i(j-k) \lambda} f^{2}(\lambda) d \lambda=\sum_{i, k=0}^{s} \frac{1}{2} t_{j} t_{k} c_{i, k}
\end{aligned}
$$

where $c_{i, k}$ is given by (44). The proof of asymptotic normality is complete by the correspondence between characteristic functions and distribution functions, for the limit of (45) as $N \rightarrow \infty$

$$
\exp \left\{-\frac{1}{2} \sum_{i, k=0}^{s} c_{i, k} t_{j} t_{k}\right\}
$$

is the characteristic function of $s+1$ jointly normal random variables with mean zero and covariances $c_{i, k}$.

Some results related to Theorem 2 are discussed in [4]. Relatively simple 
examples can be constructed to show that one need no longer have asymptotic normality if $f \notin L^{2}$ (see [7]). In fact, given any $p$ with $1 \leqq p<2$ one can construct an $f \varepsilon L^{p}$ but not in $L^{2}$ such that one does not have asymptotic normality of the covariance estimates.

\section{REFERENCES}

[1] R. Courant \& D. Hirbert, Methods of Mathematical Physics, Vol. 1, New York, 1953.

[2] U. Grenander \& G. Szegö, Toeplitz Forms and Their Applications, Berkeley, 1958.

[3] B. Grires, Uber die Spuren der verallgemeinten Toeplitzschen Matrizes, Publ. Math.Debrecen, 8 (1961), pp. 93-116.

[4] E. J. Hannan, Time Series Analysis, New York, 1960.

[5] P. MASANi \& N. WiEner, The prediction theory of multivariate stochastic processes, Acta. Math., 98 (1957), pp. 111-150.

[6] M. Rosenblatr, Asymptotic distribution of eigenvalues of block Toeplitz matrices, Bull. Amer. Math. Soc., 66 (1960), pp. 320-321.

[7] M. Rosenblatt, Independence and dependence, Proceedings of the 4th Berkeley Symposium on Probability and Statistics, 2 (1961), pp. 431-443.

Brown University

Providence, R. I. 